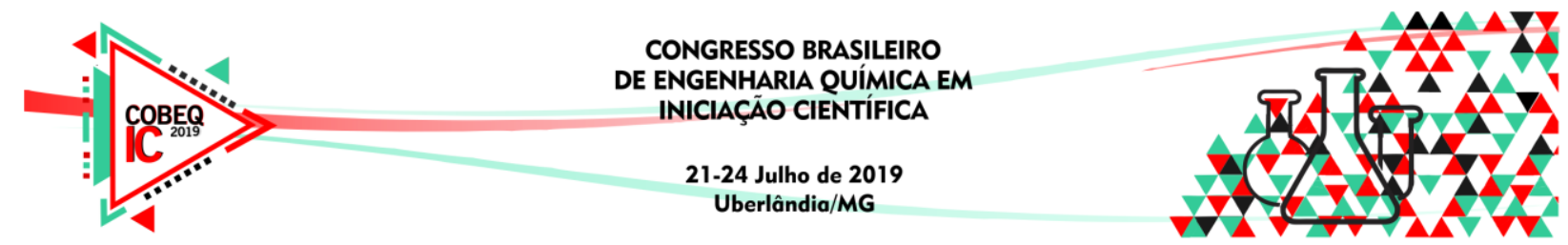

\title{
FERMENTAÇÃO ALCOÓLICA DE HIDROLISADO HEMICELULÓSICO DE TORTA DO CAROÇO DE ALGODÃO POR Galactomyces geotrichum UFVJM-R10
}

\author{
C. T. R. GARCIA ${ }^{1}$, J. P. MATOS $^{2}$, B. T. RIBEIRO ${ }^{1}$, A. S. DOS SANTOS ${ }^{3}$ e L. A. \\ PANTOJA $^{1 \text { e } 3}$ \\ ${ }^{1}$ Universidade Federal dos Vales do Jequitinhonha e Mucuri, Instituto de Ciência e \\ Tecnologia \\ ${ }^{2}$ Universidade Federal de Ouro Preto, Núcleo de Pesquisa em Ciências Biológicas \\ ${ }^{3}$ Universidade Federal dos Vales do Jequitinhonha e Mucuri, Departamento de Ciências \\ Básicas \\ E-mail para contato: camilateles13@ hotmail.com.br
}

\begin{abstract}
RESUMO - O uso da fração hemicelulósica de alguns resíduos agroindustriais para a produção de bioetanol de segunda geração (2G) depende de microorganismos capazes de fermentar pentoses e hexoses. Buscando atingir elevados valores de eficiência fermentativa e viabilizar a produção de bioetanol $2 \mathrm{G}, \mathrm{o}$ presente estudo avaliou a eficiência da produção de bioetanol a partir da fração hemicelulósica da torta do caroço de algodão utilizando a linhagem Galactomyces geotrichum UFVJM-R10. A hidrólise da biomassa foi realizada a uma razão sólido/liquido (m/v) de $31 \%$ empregando uma solução de $\mathrm{H}_{2} \mathrm{SO}_{4} 8 \%$ a $120^{\circ} \mathrm{C}$ e 1 atm de pressão por 38 minutos. Os melhores resultados de rendimento em produto $\left(1,16 \mathrm{~g} \mathrm{~g}^{-1}\right)$, eficiência fermentativa $(>100 \%)$ e produtividade volumétrica $(0,05 \mathrm{~g}$ $\mathrm{L}^{-1} \mathrm{~h}^{-1}$ ) foram observados no meio suplementado com sais minerais e nitrogênio. A partir da análise dos resultados, pode-se inferir que a linhagem G. geotirchum UFVJM-R10 possui potencial para a produção de bioetanol a partir da assimilação de glicose e xilose, sob tudo, a utilização de uma solução de nitrogênio e sais minerais deve ser levada em consideração para obter melhores resultados na produção de bioetanol $2 \mathrm{G}$.
\end{abstract}

\section{INTRODUÇÃO}

Nos últimos anos, a crescente preocupação com o meio ambiente e as oscilações no preço do petróleo estão impulsionando o interesse mundial no desenvolvimento de tecnologias sustentáveis para a produção de biocombustíveis líquidos renováveis semelhantes aos combustíveis fósseis existentes (AMARI \& KARIMI, 2018). Visando essa produção, o bioetanol lignocelulósico tem atraído a atenção da sociedade acadêmica e industrial, principalmente devido às suas vantagens econômicas e ambientais sobre outros bioprodutos orgânicos existentes (FARMANBORDAR et al., 2018). O bioetanol lignocelulósico possui um alto calor de vaporização sendo capaz de substituir a gasolina e proporcionar uma redução em até $65 \%$ da emissão dos gases de efeito estufa (DUTTA et al., 2014). Outra vantagem atribuída a este biocombustível é a sua matéria-prima, a qual é proveniente de resíduos 


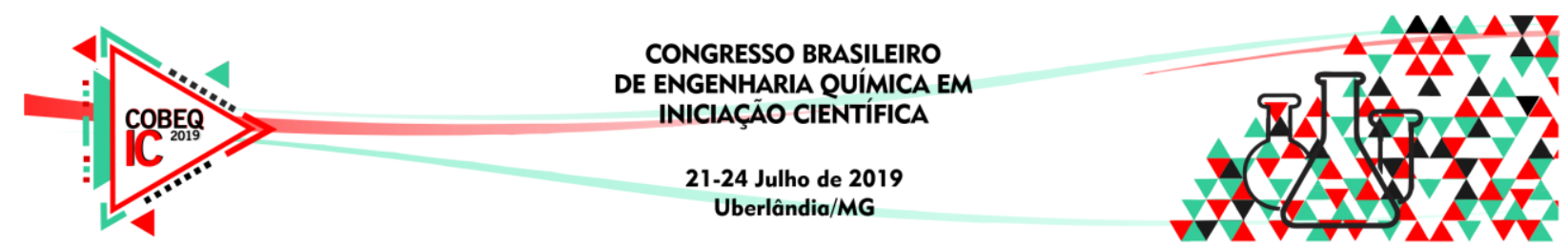

agroindustriais, o que evita a utilização de culturas alimentícias para a produção de energia (MATOS et al., 2018).

As biomassas lignocelulósicas são conhecidas por serem ricas em açúcares fermentescíveis, como pentoses e hexoses. Esses carboidratos podem sofrer fermentação alcoólica por ação de micro-organismos e apresentar o bioetanol como produto principal (AGUILAR-REYNOSA et al., 2017). A fermentação das pentoses disponibilizadas após a hidrólise da biomassa lignocelulósica, caracteriza um dos maiores desafios para a produção do bioetanol $2 \mathrm{G}$, visto que, contrariamente às hexoses, poucos micro-organismos de ocorrência natural são conhecidos como eficientes fermentadores de xilose (CADETE et al., 2016).

Em conjunto com as linhagens fermentadoras de pentoses, alguns resíduos lignocelulósicos, como a torta do caroço de algodão, foram registrados como potenciais matérias-primas para a produção do bioetanol 2G (BONISSATTO et al., 2015). Diante do potencial apresentado pela biomassa do algodoeiro na produção do bioetanol $2 \mathrm{G}$ e devido à escassez de registros do uso de G. geotrichum na fermentação alcoólica de hidrolisados hemicelulósicos, esse estudo teve por objetivo avaliar a eficiência fermentativa dessa linhagem leveduriforme na produção de bioetanol $2 \mathrm{G}$ a partir da torta do caroço de algodão.

\section{METODOLOGIA}

Obtenção e preparo da torta do caroço de algodão: A torta obtida junto à Indústria de Óleo, Rações e Plásticos Montes Claros LTDA - MG, Brasil; foi submetida à secagem em estufa com circulação forçada de ar a $60^{\circ} \mathrm{C}$ por 48 horas. Em seguida foi cominuída em moinhos de facas, peneirada em malha de $1,0 \mathrm{~mm}$, acondicionada em potes de plástico $\left(25 \pm 2^{\circ} \mathrm{C}\right)$ e reservada para uso posterior. Obtenção da fração hemicelulósica por hidrólise química: A hidrólise química foi realizada empregando uma solução de ácido sulfúrico $\left(\mathrm{H}_{2} \mathrm{SO}_{4}\right)$ a $8 \%$ e razão sólido-líquido $(\mathrm{S} / \mathrm{L})$ de $31 \%$. O ensaio foi conduzido em autoclave à $120^{\circ} \mathrm{C}$, 1 atm de pressão, por 38 minutos. Na sequência, o hidrolisado foi filtrado a vácuo sobre papel de filtro e foram recuperadas as frações insolúvel e solúvel. A fração solúvel foi neutralizado com $\mathrm{Ca}(\mathrm{OH})_{2}$ P.A e reservada para os processos fermentativos, enquanto que, a fração sólida remanescente foi submetida a sucessivas lavagens com água destilada sob filtração a vácuo em papel de filtro até pH neutro, seguida de secagem em estufa com circulação de ar forçada a $65^{\circ} \mathrm{C}$ por 24 horas e reservada para posterior caracterização físicoquímica. Preparo do inóculo: A linhagem leveduriforme G. geotrichum UFVJM-R10 foi obtida no banco de linhagens microbianas do Laboratório de Bioprocessos e Biotransformação da UFVJM. A linhagem foi reativada em meio YMPD (Yeast Malt Peptone Dextrose), e incubadas a $28{ }^{\circ} \mathrm{C}$ por 48 horas. O inóculo foi preparado a partir de uma alçada da linhagem reativada em $100 \mathrm{~mL}$ do meio descrito por Toquero e Bolado (2014). As culturas cresceram em frascos cônicos de $250 \mathrm{~mL}$ incubados a $28^{\circ} \mathrm{C}$ sob agitação de $150 \mathrm{rpm}$ por tempo suficiente para se atingir 1 unidade de densidade óptica (D.O) com leitura espectrofotométrica a $600 \mathrm{~nm}$. Após o período de crescimento, o meio foi centrifugado por 15 minutos a $4000 \mathrm{rpm}$ e o sobrenadante foi descartado. As células foram lavadas com água destilada estéril, ressuspensas finalmente em $100 \mathrm{~mL}$ de água estéril e, nessa forma, usadas como inóculo na proporção de $10 \%$ v/v. Fermentação alcoólica: O ensaio fermentativo foi conduzido em frascos cônicos de $50 \mathrm{~mL}$ contendo o hidrolisado hemicelulósico com e sem 


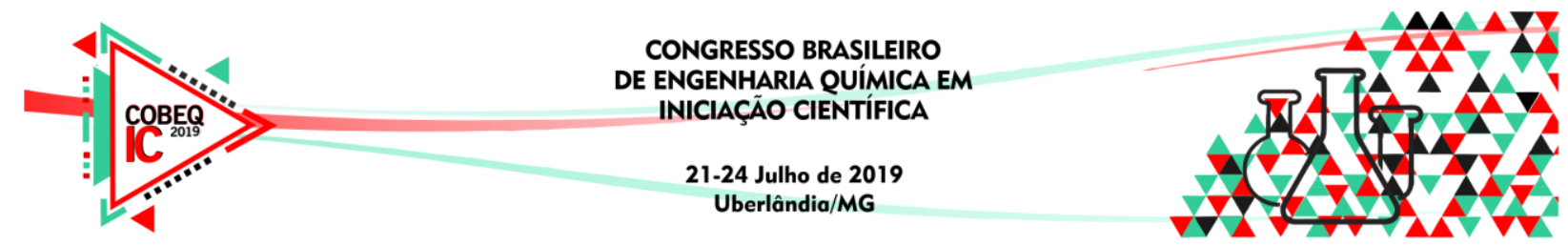

suplementação de sais minerais. O hidrolisado suplementado recebeu a adição do mesmo meio de cultivo usado para o preparo do inóculo, sem a presença de glicose e xilose, na proporção de 1:1. Alíquotas de $1 \mathrm{~mL}$ foram colhidas a intervalos de 4 horas e centrifugadas a $15.000 \mathrm{rpm}$ por 5 minutos. Os sobrenadantes foram armazenados a $-20^{\circ} \mathrm{C}$ para determinações analíticas posteriores e as células foram resuspensas a $1 \mathrm{~mL}$ com água destilada para determinação da D. $0_{600 \mathrm{~nm}}$. Os processos fermentativos foram avaliados ainda, quanto aos parâmetros de rendimento em etanol; produtividade volumétrica de etanol e eficiência fermentativa (HISS, 2013). Caracterização físico-química da torta antes e após o prétratamento ácido: A torta foi caracterizada quanto aos teores de fibra em detergente neutro FDN, fibra em detergente ácido - FDA, celulose, lignina, hemiceluloses (AOAC, 1992) e teor de amido (McCREADY et al.,1950). Todas as análises foram realizadas em triplicata.

\section{RESULTADOS E DISCUSSÃO}

A torta do caroço de algodão analisada nesse estudo apresentou $11 \%$ de hemiceluloses, $23 \%$ de celulose e $4,5 \%$ de amido (Tabela 1 ). Esses carboidratos em conjunto somam 38,5\% do peso seco da torta do caroço de algodão, o que revela o seu potencial para a produção de bioetanol.

Tabela 1 - Composição química da torta do caroço de algodão antes e após tratamento químico com $\mathrm{H}_{2} \mathrm{SO}_{4}$ a $8 \%$

\begin{tabular}{ccc}
\hline \multirow{2}{*}{ Frações (\%) } & \multicolumn{2}{c}{ Biomassa } \\
\cline { 2 - 3 } & Antes do pré-tratamento & Após o pré-tratamento \\
\hline Amido & $4,50 \pm 0,74$ & ND \\
Celulose & $23,36 \pm 1,23$ & $42,98 \pm 2,67$ \\
Hemicelulose & $11,26 \pm 0,31$ & $4,78 \pm 1,14$ \\
Lignina & $6,94 \pm 1,58$ & $28,96 \pm 1,44$ \\
\hline
\end{tabular}

O valor de $6,9 \%$ de lignina encontrado na torta estudada (Tabela 1) pode ser considerado baixo para biomassas lignocelulósicas. Os baixos teores de lignina favorecem o uso da biomassa para produção de etanol $2 \mathrm{G}$, uma vez que a lignina tem a função de aumentar a resistência da estrutura lignocelulósica a ataques químicos e enzimáticos, por consequência, quanto menor teor desse componente, menor será a resistência para liberação dos açúcares passíveis para a fermentação (SANNIGRAHI et al., 2011). A lignina (28\%) e a celulose (42\%) após o pré-tratamento ácido foram concentradas no resíduo sólido remanescente (Tabela 1). As taxas relativamente mais elevadas desses carboidratos, em biomassas prétratadas com ácido sulfúrico, podem estar relacionadas à perda concomitante de polissacarídeos (hemiceluloses), que ao serem removidos pelo reagente utilizado, promove o aumento da concentração da fração que fica retida no resíduo sólido restante (MATOS, 2018).

Quanto ao perfil de crescimento, G. geotrichum obteve valores iniciais de aproximadamente 1 unidade até valores próximos entre 20 e 24 unidades de D. O600nm no $_{6}$ hidrolisado puro e suplementado, respectivamente, em 96 horas de fermentação (Figura 1). Em ambos os meios, o micro-organismo apresentou um perfil de crescimento semelhante, indicando que a suplementação com sais e nitrogênio não interferiu no desenvolvimento da levedura. 


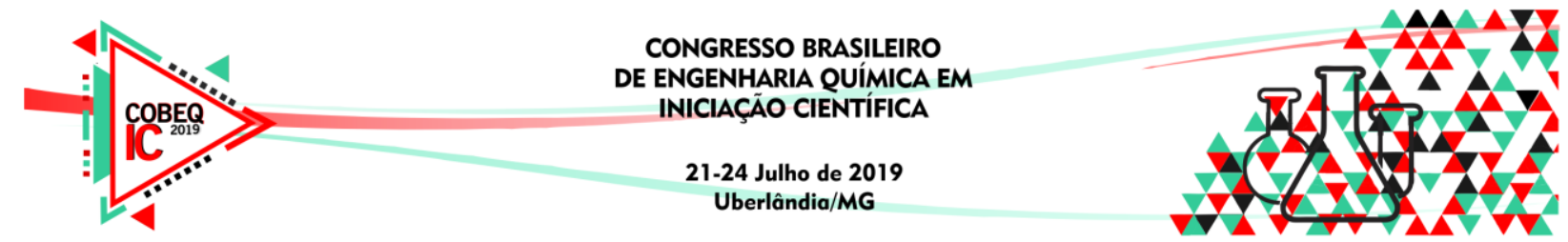

Figura 1 - Perfil do crescimento celular da linhagem Galactomyces geotrichum UFVJM-R10

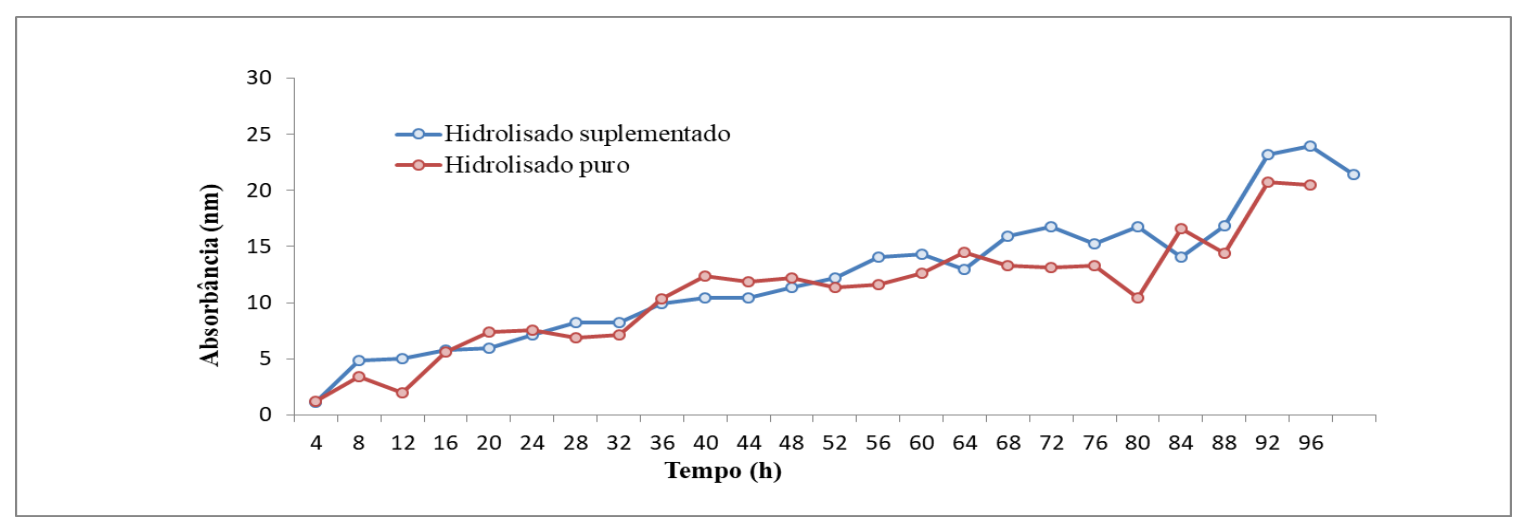

O perfil do consumo de glicose por G. geotrichum UFVJM-R10 no meio suplementado com a solução de sais minerais e nitrogênio (Figura 2A) foi observado em 20 horas de fermentação, entretanto, no meio não suplementado (Figura 2B) a glicose foi totalmente consumida a partir das 60 horas de fermentação e assim como a glicose, no meio suplementado, o consumo total de xilose foi mais rápido quando comparado ao consumo desse mesmo açúcar no meio não suplementado, cujo carboidrato manteve-se presente até o fim da fermentação.

Figura 2 - Perfil do consumo de açúcares e produção de etanol por G. geotrichum UFVJMR10 em hidrolisado puro (A) e suplementado com sais minerais (B)
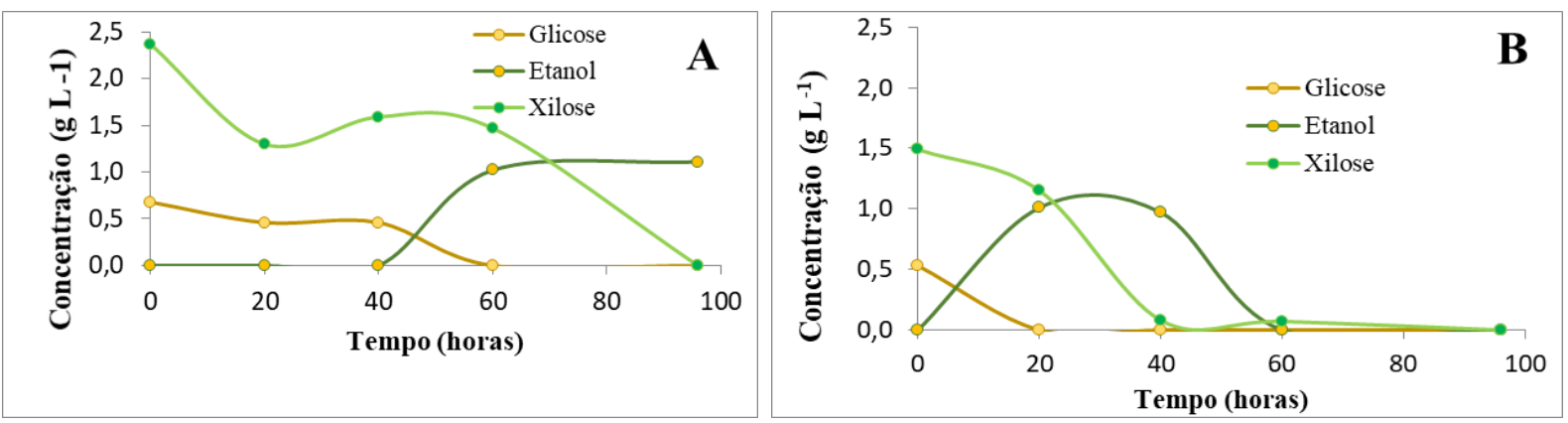

A suplementação de um meio fermentativo tem por objetivo melhorar o desempenho de leveduras e aumentar a sua produtividade volumétrica (DANESI, 2009). E nesse estudo, pode-se observar que a suplementação proporcionou um melhor desempenho da levedura quanto à metabolização dos açúcares presentes no meio. Além disso, sabe-se que açúcares de cinco carbonos, como a xilose, não são facilmente fermentados por qualquer linhagem de micro-organismos e muitas vezes quando são assimilados, ocorre de forma mais lenta que a glicose (BERŁOWSKA et al., 2016), o que pode explicar o consumo mais acelerado da glicose em relação ao de xilose nos dois meios estudados.

Os parâmetros fermentativos do processo foram calculados considerando os tempos onde houve a melhor produção de etanol (Tabela 2), porém, a xilose, no meio não suplementado, não havia sido consumida por completo. A maior produção de etanol obtida pela levedura estudada no meio não suplementado $\left(1,11 \mathrm{~g} \mathrm{~L}^{-1}\right)$ e suplementado $\left(1,01 \mathrm{~g} \mathrm{~L}^{-1}\right)$ ocorreu após 96 e 20 horas de fermentação, respectivamente (Tabela 2). Em ambos os casos, a maior concentração de etanol presente nos meios adveio após o consumo total da glicose. Os 


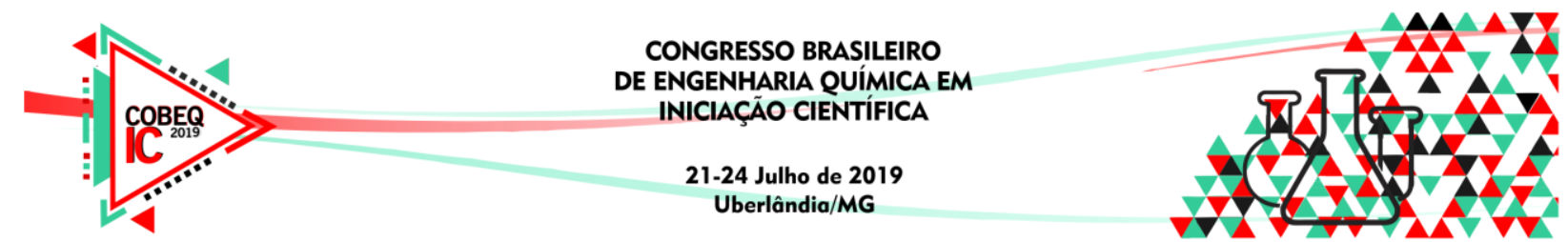

intervalos de tempo observados para o maior rendimento em etanol em ambos os meios analisados, mostram que a suplementação, além de aumentar a produção, o faz em menos horas quando comparado ao meio sem suplementação. Industrialmente, a otimização em relação ao tempo de produção pode significar economia e elevação de lucro e produção.

Em relação ao rendimento em produto e eficiência fermentativa os valores no meio suplementado foram mais expressivos $\left(1,16 \mathrm{~g} \mathrm{~g}^{-1}\right)$ e $(>100 \%)$, respectivamente, quando comparados ao meio sem suplementação $\left(0,36 \mathrm{~g} \mathrm{~g}^{-1}\right)$ e $(71,22 \%)$, respectivamente. Além disso, a suplementação de sais ao ensaio contribuiu para o aumento da produtividade volumétrica $\left(0,05 \mathrm{~g} \mathrm{~L}^{-1} \mathrm{~h}^{-1}\right)$ (Tabela 2). Esses valores mostram que a adição de fontes de sais e nitrogênio ao meio acelera a produção máxima de etanol por G. geotrichum UFVJM-R10, bem como, favorece a elevação da produtividade e eficiência fermentativa.

Tabela 2 - Processos fermentativos conduzidos com G. geotrichum UFVJM-R10 no hidrolisado hemicelulósico suplementado e não suplementado

\begin{tabular}{cc|c}
\hline \multirow{2}{*}{ Parâmetros de Processo } & \multicolumn{2}{c}{ Hidrolisado } \\
\cline { 2 - 3 } & Não suplementado $(96 \mathrm{~h})$ & $\begin{array}{c}\text { Suplementado com sais minerais } \\
(20 \mathrm{~h})\end{array}$ \\
\hline Etanol $\left(\mathrm{g} \mathrm{L}^{-1}\right)$ & 1,11 & 1,01 \\
$\mathrm{Y}_{\mathrm{P} / \mathrm{S}}\left(\mathrm{g} \mathrm{g}^{-1}\right)$ & 0,36 & 1,16 \\
$\mathrm{Q}_{\mathrm{P}}\left({\left.\mathrm{g} . \mathrm{L}^{-1} \mathrm{~h}^{-1}\right)}^{\mathrm{E} f(\%)}\right.$ & 0,01 & 0,05 \\
& 71,22 & $>100$ \\
\hline
\end{tabular}

\section{CONCLUSÃO}

A levedura Galactomyces geotrichum UFVJM-R10 apresentou capacidade de produção de etanol a partir do hidrolisado hemicelulósico da torta do caroço de algodão, assimilando tanto glicose como xilose. A suplementação, de sais minerais e nitrogênio no meio fermentativo, possibilitou uma elevada eficiência $(>100 \%)$ fermentativa e reduziu o tempo de fermentação (em 76 horas). Os resultados obtidos mostram que a linhagem G. geotrichum possui potencial para a produção de bioetanol. No entanto, há necessidade de otimização dos processos para melhorar a produção de etanol e aumentar a produção do produto de interesse.

\section{REFERÊNCIAS}

AGUILAR-REYNOSA, A.; ROMANÍ, A.; RODRÍGUEZ-JASSO, R. M.; AGUILAR, C. N., GARROTE, G.; RUIZ, H. A. Microwave heating processing as alternative of pretreatment in second-generation biorefinery: An overview. Energ Convers Manage, v. 136, p. 50-65, 2017.

AMARI, H. \& KARIMI, K. Pretreatment and hydrolysis of lignocellulosic wastes for butanol production: Challenges and perspectives. Bioresource Technol., 2018.

ASSOCIATION OF OFFICIAL ANALYTICAL CHEMISTRY - AOAC. Official methods of analysis of the Association of Official Analytical Chemistry. Washington: AOAC, 1992. 


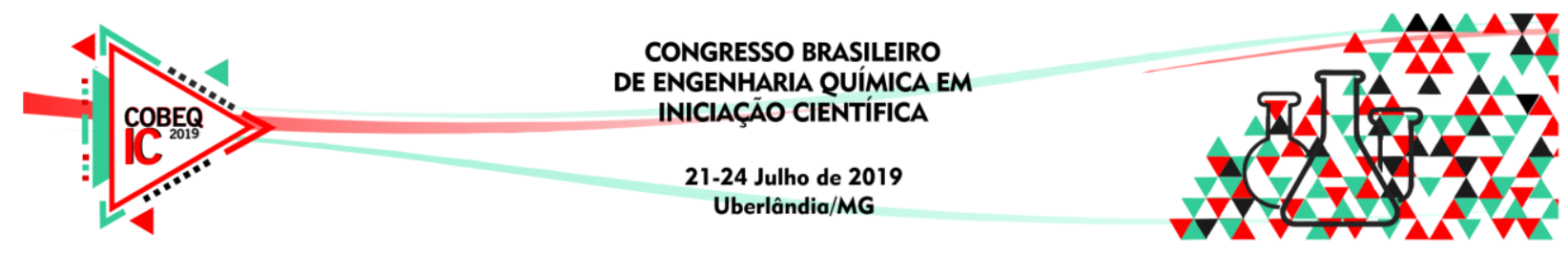

BERŁOWSKA, J.; PIELECH-PRZYBYLSKA, K.; BALCEREK, M.; DZIEKO`NSKAKUBCZAK, U.; PATELESKI, P.; DZIUGAN, P.; KREGIEL, D. Simultaneous saccharification and fermentation of sugar beet pulp for efficient bioethanol production. BioMed res int, v. 2016, 2016.

BONISSATTO, R. C.; D.J. SILVA, J. M. de A.; RODRIGUES, F. A.; RESENDE, S. T.; de MENDONÇA NETO, A. B. Processo de produção de etanol a partir da hidrólise ácida de biomassa lignocelulósicas. Blucher Chem. Engi. Proceedings, v. 1, n. 2, p. 2370$2377,2015$.

CADETE, R. M.; ALEJANDRO, M.; SANDSTRÖM, A. G.; FERREIRA, C.; GÍRIO, F.; GORWA-GRAUSLUND, M. F.; ROSA, C. A. FONSECA, C. Exploring xylose metabolism in Spathaspora species: XYL1. 2 from Spathaspora passalidarum as the key for efficient anaerobic xylose fermentation in metabolic engineered Saccharomyces cerevisiae. Biotechnol Biofuels, v. 9, n. 1, p. 167, 2016.

DANESI, E.D.G.; MIGUEL, A.S.M.; RANGEL-YAGUI, C.O.; CARVALHO, J.C.M.; PESSOA Jr., A. (2006). Effect of C:N ratio and substrate source on glucose-6phosphate dehydrogenase (G6PDH) production by recombinant Saccharomyces cerevisiae. J Food Eng., v. 75, p. 96-103, 2009.

DUTTA, K.; DAVEREY, A.; LIN, J. Evolution retrospective for alternative fuels: First to fourth generation. Renew Energ, v. 69, p.114-122, set. 2014.

FARMANBORDAR, S.; KARIMI, K.; AMIRI, H. Municipal solid waste as a suitable substrate for butanol production as an advanced biofuel. Energy Conversion and Management, v. 157, p. 396-408, 2018.

HISS, H. (Ed.). Cinética de Fermentações: Uma análise matemática da atividade microbiana. São Paulo: Edição do Autor, 2013. 654p. ISBN 98785915695.

MATOS, J. P.; SOUZA, K. R.; SANTOS, A. S.; PANTOJA, L. A. FERMENTAÇÃO ALCOÓLICA DE HIDROLISADO HEMICELULÓSICO DE TORTA DE GIRASSOL POR Galactomyces geotrichum UFVJM-R10 E Candida akabanensis UFVJM-R131. Quím Nova, v. 41, n. 1, p.23-2018.

MCCREADY, R. M., GUGGOLZ, J., SILVIERA, V., \& OWENS, H. S. Determination of starch and amylose in vegetables. Application to pear. Anal Chem., v.22, p. 1156, 1950.

SANNIGRAHI, P., KIM, D. H., JUNG, S., \& RAGAUSKAS, A. Pseudo-lignin and pretreatment chemistry. Energ Environ Sci., v. 4, n. 4, p. 1306-1310, 2011.

TOQUERO, C. \& BOLADO, S. Effect of four pretreatments on enzymatic hydrolysis and ethanol fermentation of wheat straw. Influence of inhibitors and washing. Bioresource Technol, v. 157, p. 69-76, 2014. 